\title{
ADVANCED POSITIONING AND LOCATION BASED SERVICES IN 4G MOBILE-IP RADIO ACCESS NETWORKS
}

\author{
Alberto Montilla Bravo', José Ignacio Moreno ${ }^{2}$, Ignacio Soto $^{3}$ \\ ${ }^{1}$ Motorola España S.A., C/ Martinez Villergas 52 Bloque 3, 28027, Madrid, Spain, alberto.montilla@motorola.com \\ ${ }^{2,3}$ Departamento Ingeniería Telemática, Universidad Carlos III de Madrid, Av. De la Universidad 30, E-28911 Leganés
} (Madrid), Spain, ${ }^{2}$ joseignacio.moreno(aieee.org, ${ }^{3}$ isoto@it.uc3m.es

\begin{abstract}
This paper proposes an evolved architecture from 3G networks to provide basic and advanced positioning methods for Location Based Services in Mobile lPv6-based Radio Access Networks. We start analyzing current status of Location-Based Services (i.e. LBS or LCS) and architectures in $3 \mathrm{G}$ networks as well as state-of-the-art research on LBS and Mobile Internet. Next we set the requirements the solution should fulfill. We continue proposing the evolved architecture for support of basic and advanced positioning methods, using MIPv6 and HMIPv6 as mobility scenario for the Mobile IPv6 based RAN, describing element's functions and changes from current approaches as well as description of the dynamic behavior. We complete the proposal with a bandwidth analysis of the signaling, identifying issues when planning implementation of LCS services in the network.
\end{abstract}

Keywords - 4G, Positioning, LBS, LCS, MIPv6, Radio Access Networks.

\section{INTRODUCTION}

Most of current mobile systems provide support for basic positioning methods that allow basic Location Based Services. Examples of these methods are location based on Cell Identifier and Timing Advance, which provide location estimation with error of hundreds of meters, giving support for a limited set of services (e.g. find the nearest Gas station) but are not suitable for advanced location services (e.g. estimate the route to find a store in a shopping center). In $2.5 \mathrm{G}$ and $3 \mathrm{G}$ mobile systems, advanced positioning methods have been introduced to provide support for a full set of advanced location based services; those methods include but are not limited to:

- Delay measurement on cell's downlink signals (includes AFLT-cdma2000, OTDOA-UMTS and EOTD-GSM), based on cell's delay measurements performed at the Mobile Station and assistant measurement units, providing a location estimation with high accuracy.

- Assisted GPS (GSM, UMTS and cdma2000), based on a GPS receiver equipped in the Mobile Station and GPS assistance data provided by the network to help satellite signal acquisition, providing location estimation with high accuracy.
In order to support these methods, some elements were added to the $2.5 \mathrm{G} / 3 \mathrm{G}$ network architecture, which are shown in Figure 1:

- General LCS server (GMLC-UMTS and MPCcdma2000): The LCS server is the gateway from where the LBS clients can obtain the user's location. It is the interface towards location in the mobile system.

- Positioning (i.e. Measurements and calculation) units (SMLC and LMU- UMTS and PDE-cdma2000): There are two units involved in positioning, one responsible to collect radio signal measurements (LMU-UMTS and LPDE-cdma2000) for the different positioning methods, that together with the Mobile station measurements are reported to the calculation unit (SMLC-UMTS and PDE-cdma2000) which is responsible for calculating the position of the user depending on method and measurements.

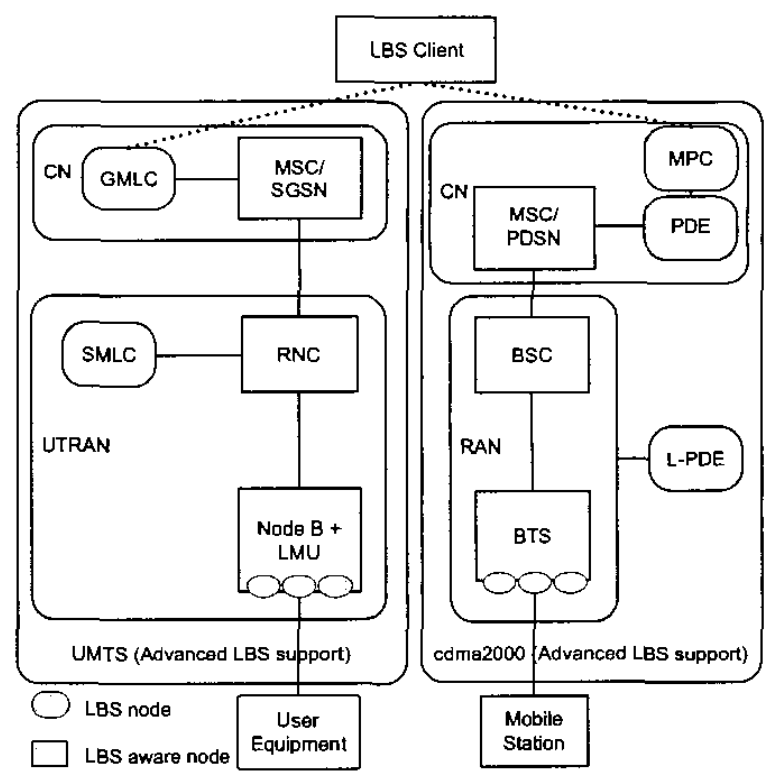

Fig. 1. LBS network architecture in $3 \mathrm{G}$ networks.

With these network elements it is possible to locate the user under any scenario with high accuracy, thus allowing the 
implementation of Advanced Location Based Services that need such accuracy.

Current trends in IETF, other standardization forums and general research for $4 \mathrm{G}$ Mobile networks include the support for Mobile IPv6 (MIPv6) as main mobility IP-based architecture as well as the support of Hierarchical Mobile IPv6 (HMIPv6) for mobility architecture in the Radio Access Network (RAN) [1]. These architectures and its associated protocols provide support form identification, mobility, handover, paging, etc, which provides continuation of current network services over the Mobile Internet. However, the standardization bodies have not addressed the support of advanced positioning methods in such architectures.

In [2], it is proposed the support of basic LBS services in MIPv6. This proposal is based on the use of Binding Update to obtain the positioning information from the Access Point ID in WLAN/Bluetooth, the two tcchnologies considered in the study. The proposal emphasizes it is not suitable for indoor scenarios due to the location accuracy error of the Access Point ID approach, similar to Cell-ID in cellular systems. In addition, the proposal is mobile-triggered in general case (one may think of forcing periodical-andfrequent sending of Binding Update, however it would have big impact on signaling bandwidth).

In [3], the author analyzed the current 3G positioning methods for the existing specified $3 G$ systems, and recommended $3 \mathrm{GPP}$ and $3 \mathrm{GPP} 2$ (bodies in charge of technical specification of UMTS and cdma2000 respectively) start working jointly towards the harmonization of $3 \mathrm{G}$ positioning protocols and interfaces, due to the commonality of functions and information exchanges, especially in those cases the positioning method is not directly related to the Radio Access Technology used.

The Geopriv working group of IETF, is working towards the specification of the host location information transferred over Internet, and drafted in [4] the requirements for the transport of location information over Internet, through the use of a Location Object (LO) containing the location information, and four logical entities:

- Location Generator (LG), which obtains the user's location, creates the LO and publish it. The document does not specify how the user's location is obtained,

- Location Server (LS) that receives announcements and subscriptions requests from LBS clients. It applies the rules to the received LO and sends it to the appropriate clients,

- Location Receiver (LR), which is the client receiving the Location Object, and

- Rules Holder (RH), which maintains the rules for reception, filtering and distribution of location objects.
The Geopriv proposal shows an example scenario of a cellular/mobile network, where the LG function is located at the border of the Mobile network (typically a GMLC or MPC). In this scenario there is no use of the LO inside the mobile network. In addition, the solution does not specify any protocol for exchanging/transferring the LO, neither specifies methods for different types of requests (e.g. periodic, event-based), because it is intended to provide security and privacy for the location information as main goal.

In mobile networks that typically provide support for authentication, integrity protection and ciphering, the advantages of including support of Geopriv LO are questionable, because main advantages added by Geopriv are already supported $i m$ the system. In the Internet, the scenario is different, as networks and clients are not controlled as in a mobile network, including fixed and mobile nodes that are distributed across the globe. In this case the use of Geopriv LO is valuable as it is extending security of location information to a typical non-secure context.

\section{REQUIREMENTS OF LBS SUPPORT IN 4G RADIO ACCESS NETWORKS}

\section{A. General requirements}

The LBS support in Mobile IP RAN, requires the support of positioning determination services towards the Core Network for Advanced positioning methods. This imposes following requirements:

- The proposed LBS architecture in the Mobile IP RAN should provide positioning determination services to the Core Network LBS network elements, which provides the LBS services to end-users (LBS clients).

- The proposed LBS architecture in the Mobile IP RAN should provide support to the positioning methods defined in GSM, IS-95, UMTS and cdma2000 at minimum (see [5] and [6]), and should be open to introduce new positioning methods as they become available.

- The proposed LBS architecture should support positioning calculation that provide positioning in the shapes defined by $3 \mathrm{GPP}$ and $3 \mathrm{GPP} 2$ at minimum (see [6] and [7]), and shall be open to introduce new geographical shapes as they become available.

\section{B. Network and Protocols}

The LBS support in Mobile IP RAN is implemented by Location-based protocols and entities; protocols are required to operate transparently over the Mobile IP network and generic to the Radio Technology used by the Mobile Station. 
- The LBS architecture and protocols in Mobile IP RAN should be generic to the Radio Access Technology used (i.e. it is not desired the protocols to be specific to the technology used, as one of the main goals of integrating the LBS in Mobile Internet is the harmonization of the LBS protocols across technologies).

- The LBS architecture and protocols should have minimum or no impact over the Mobility protocols, i.e. MIPv6 and HMIPv6, and over its associated nodes, i.e. Home Agent (HA) and MAP (Mobility Anchor Point).

\section{Support for external networks (LBS clients)}

- The Mobile IP-based Mobile Network (including RAN and Core Network), should provide support for traditional LBS clients, based on Legacy location protocols, e.g. [8] and to Internet clients, based on use of Georpriv Location Object [4], in order to ensure evolution of network whist maintaining connectivity to legacy clients.

\section{MOBILE-IP LBS ARCHITECTURE}

In order to meet the above requirements, it is proposed a LBS architecture as an evolution of current $3 \mathrm{G}$ architectures, suitable for use in $4 \mathrm{G}$ Mobile IP-based networks. Figure 2 shows the proposed architecture, including nodes functions providing support for basic and advanced positioning determination services:

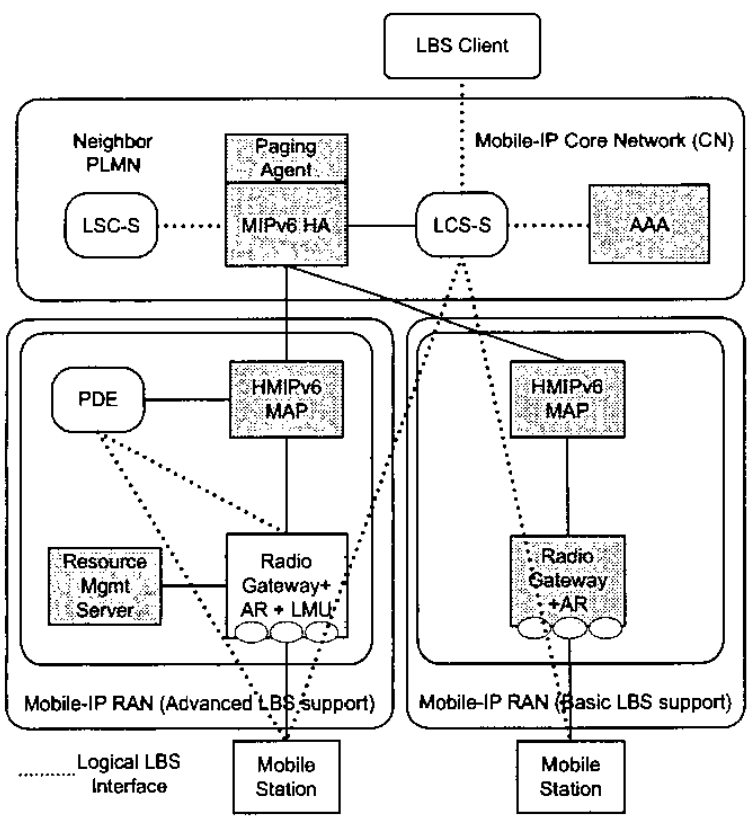

Fig. 2. Mobile-IP RAN LBS proposed architecture.

1. The Basic Location architecture (rightmost in figure) determines positioning based on Cell Identity (Access point ID) or based completely on Mobile Station features e.g. Stand-alone GPS. For this level of LBS support, there is no need for new elements in the network other than the Location Server (LCS-S).

2. The Advanced Location architecture (Leftmost in figure) determines positioning based on any method existing in $3 \mathrm{G}$ and those adapted to new access technologies like Bluetooth or WLAN.

\section{A. Core Network}

In the Core Network, the Mobile-IP LBS architecture is composed by the Location Services Server (i.e. LCS-S). The LCS-S is responsible for communicating with the LBS clients through a standard interface, either [8] or [4]. The LCS-S is responsible for the management of LBS clients (i.e. AAA in conjunction with the AAA network node, coordination of location requests, coordinates transformation, etc.). It also communicates with the Mobile Station to request its position, through a proposed new interface based on [8]. The LCS-S announces its services to the Mobile Stations as well to the positioning elements by using a Service Location protocol [9]. Note LCS-S announcements are needed because the support network (i.e. Mobile IP) is not aware of any Location Information, which was not the case of MSC/SGSN or MSC/PDSN in 3G networks. The LCS-S is also in charge of managing Location requests due to emergency or Legal Interception entities.

\section{B. Mobile-IP RAN}

In the Radio Access Network, the Mobile-IP LBS architecture is composed by the following network elements:

- Position Determination Entity (PDE), which main function is the calculation of the position by using positioning algorithms based on measurements taken by the Mobile Station and Location Measurement Units (LMU) or by using the network location information (e.g. Cell Identifier). The PDE contains algorithms for each of its supported positioning methods. Its functionality is similar to the provided by the SMLC in UMTS or PDE in cdma2000. Main difference is that its protocols are adapted and harmonized to a generic Radio Access Technology, so that they can be used by any specific Radio Access Technology. Similar to the LCS-S the PDE maintains communication with the Mobile Station to exchange measurement information. Because of that, the PDE must announce it services to the Mobile Stations using same protocol [9] as LCS-S does. In these advertisements the PDE announces the positioning methods supported so that the Mobile Stations can adapt their requests to the positioning methods supported by the PDE.

- Location Measurement Unit (LMU), which performs measurements over the radio interface to provide $\mathrm{PDE}$ 
with enough data for an accurate positioning estimation. Note this element might be integrated in the Radio Gateways of the system, as it needs to support the Radio Access Technologies over which it performs measurements. The LMU maintains communications with the PDE and announces its services to the PDEs in the RAN by using [9].

C. Modifications and impacts on the Mobile IP based network elements

- AAA: Changes in AAA servers are limited to the support of location register in the user profile Data Base. AAA is in charge of providing the authentication and authorization information to the LCS-S. This register contains similar info to current HLR LCS register in $3 \mathrm{G}$ networks.

- Mobility nodes (i.e. HA, PA and MAP): The HA, MAP and PA do not need any modification to support the LBS architecture proposed here.

- Radio Gateway: The Radio Gateway comprises the functions of a Radio Base Station and IP Access Router in the Mobile IP RAN. To support LBS services, the RG IP-Radio Access Technology middleware implements a function to map the LCS-S and PDE announcements to broadcast system information delivered to the users. The mapping is specific to each Radio Access Technology and it is performed for efficiency purposes.

- Mobile Station: The MS behavior changes with respect to its $3 \mathrm{G}$ behavior. The MS is in charge of coordinating the request in the RAN serving as bridge between the LCS-S (CN) and the PDE (RAN). This is done with the purpose of maintaining the Mobile IP network nodes unaware of the location services in order to avoid impacting the scalability of the LBS proposal and avoiding complicating the operation of HA and MAP.

\section{ANALYSIS}

In order to validate properly the proposal, there were two analyses performed, first an analysis of the dynamic behaviour of the system by the creation and study of Message Sequence Charts with the purpose of determining possible communication problems (e.g. race conditions or timing problems), and second a Bandwidth analysis of the signalling exchanged between the elements and its constraints.

\section{A. Dynamic Behaviour analysis}

Message Sequence Charts - MSC were created for several scenarios, i.e. Client initiated LBS positioning request - UE in IDLE state, Client initiated LBS positioning request - UE in ACTIVE state, Mobile Station initiated LBS positioning request - UE in ACTIVE state and Mobile Station initiated
LBS positioning request - UE in IDLE state. Figure 3 shows the dynamic behaviour of the system in the Client initiated LBS positioning request when user is in ACTIVE state:

- The LBS client issues a Location Immediate Request message to the LCS-S indicating the address (E.164 number of IP home address) of the target.

- The LCS-S authorizes client towards AAA and routes the message towards the HA.

- The HA, and MAP redirect the Location Immediate Request message towards the user by standard MIPv6 and HMIPv6 mechanisms, reaching the MS. Note in case the MS is in IDLE state, the PA, before redirecting the message, issues a Paging to the user, establishing radio link, performs Binding Updating, authenticating the user and providing privacy to the data.

- The MS after receiving the request, issues a Position Request message to the PDE indicating its supported methods, the Radio Technology used as well as the required accuracy. With this information, the PDE selects the positioning method and require the MS and the LMU to perform measurements, which are back reported to the PDE. Then PDE calculates the position which is sent to the MS in the Position Report message.

- The MS stores the position and sends it to the LCS-S in the Location Immediate Report message. LCS-S stores the Location (it may translate coordinates) and sends the Location to the client.

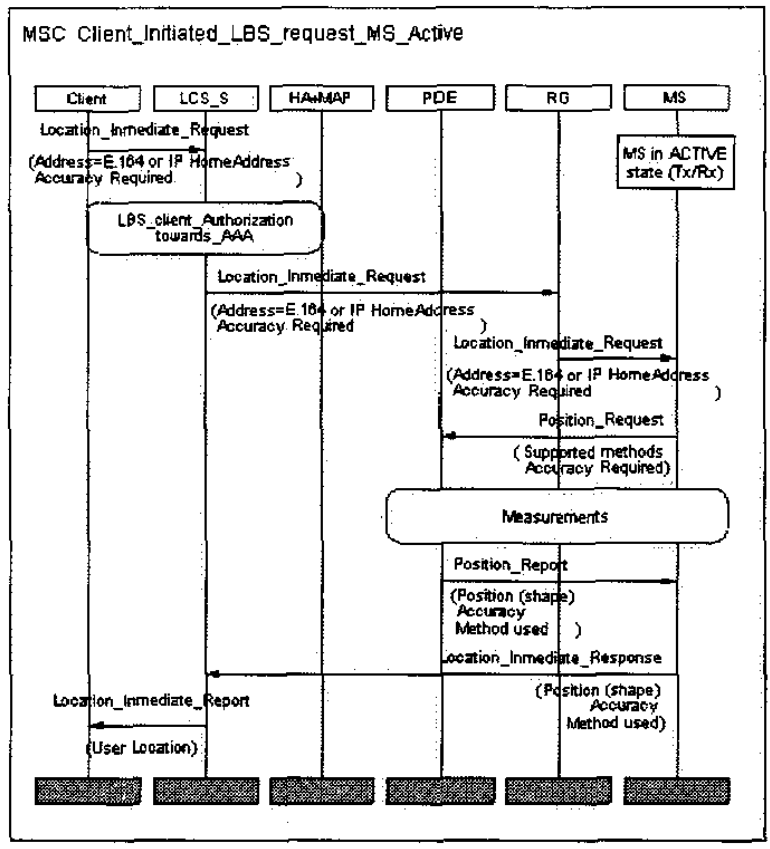

Fig. 3. Client initiated LBS positioning request, user in ACTIVE state. 


\section{B. Bandwidth analysis in the Radio Interface}

In order to determine bandwidth consumption due to basic and advanced positioning, it was estimated the bandwidth usage in the Radio Access due positioning requests, because it is the bottleneck of the system.

To do this analysis we assumed a UMTS DCCH logical channel carried over common transport channels (RACH/FACH), of rate $32 \mathrm{kbps}$. Table 1 shows the considered messages. Those message sizes are at UMTS MAC layer (RACH/FACH), i.e. includes transport (UDP) and IPv6 related headers.

\section{Table 1}

LBS-related messages in Mobile IP RAN (Air interface).

\begin{tabular}{|l|c|c|}
\hline \multicolumn{1}{|c|}{ Message } & Direction & $\begin{array}{c}\text { Size (bits } \\
\text { MAC) }\end{array}$ \\
\hline Location Immediate Request & MS & 1008 \\
\hline Location Immediate Report & LCS-S & 1680 \\
\hline Position Request & PDE & 832 \\
\hline Position Report & MS & 1008 \\
\hline Measurement Request & MS & 672 \\
\hline Measurement Response & PDE & 1176 \\
\hline
\end{tabular}

Equation 1 defines the bandwidth consumption in the Radio Interface due to the proposed LBS dynamic behavior:

$$
\mathrm{BW}_{\mathrm{LCS}}=\mathrm{N}_{\text {user }} \times \mathrm{LOC}_{\mathrm{Freq}} \times \sum_{\text {LCS_messages }} \text { Message_size }
$$

$\mathrm{BW}_{\mathrm{LCS}}$ is the Bandwidth used by LBS positioning requests; $\mathrm{N}_{\text {user }}$ is the number of users in the channel; LOC $_{\mathrm{Freq}}$ is the LBS positioning Request frequency, in 1/sec; Message_size is the bit size of each message (LCS messages).

Bandwidth was calculated for frequencies from 0.0001 to 0.001 (from 0.36 to $3.6 \mathrm{LBS}$ requests/hour), with 1 to 500 users per cell. Results showed the LBS position requests used low bandwidth for all cases described (e.g. bandwidth is $1.85 \mathrm{kbps} @ 3.6$ Location Request/hour and 500 users). Figure 4 shows the occupancy of the $32 \mathrm{kbps}$ RACH/FACH channel, which shows a low level of utilization (e.g. utilization is $5.8 \%$ with 500 users @ 3.6 LBS requests/hour each).

Results showed also that Bandwidth consumptions depend linearly on the frequency of the positioning requests. This aspect shall be taken into account when designing and planning the deployment of LBS over the Mobile-IP based RAN (and other type of network as it is common to all technologies) in order not to compromise other services. Possibilities include e.g. defining services without the need of immediate location so that the LCS-S can provide the client with an aged position estimated previously, so that the request is not passed to the Mobile-IP RAN.

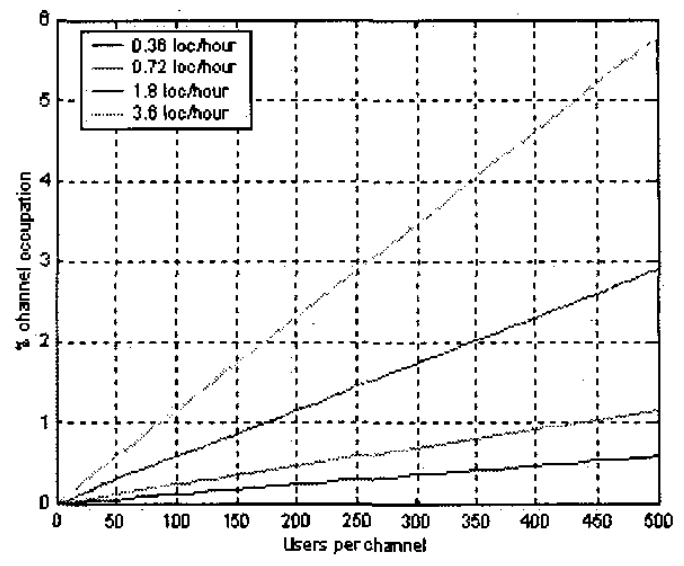

Fig. 4. BW utilization of LBS requests vs. number of users in a cell (assumed UMTS 32kbps RACH/FACH DCCH).

\section{CONCLUSIONS}

This paper presented a proposal for the support of advanced positioning and Location Based services over 4G Mobile-IP Radio Access Networks, based on the evolution of existing LBS services to a Mobile-IP based architecture. It described the architecture and the logical elements that compose it, as well as an analysis based on dynamic behavior and Bandwidth usage that validated the architectural approach, showing low bandwidth consumption under common scenarios.

\section{REFERENCES}

[1] A. Montilla, J. Moreno and I. Soto. "Mobile-IP RAN: Arquitectura IP Móvil en Redes de Acceso Radio 4G" (in Spanish). XIII National Telecom R\&D, ISBN 8489315-28-0. November 20, 2003.

[2] S. Thongthammachart and H. Olesen. "Mobile location services over the next generation IP core network". IEEE $10^{\text {th }}$ international Conference on Telecommunications. Volume I. ICT 2003, pp. 324-329. March 1, 2003.

[3] Y. Zhao. "Standardization of Mobile Phone Positioning for $3 \mathrm{G}$ Systems". IEEE Communications Magazine, July 2002. pp. 108-116.

[4] J. Cuellar et al. "Geopriv requirements". draft-ietfgeopriv-reqs-04.txt (work in progress), October 2003.

[5] 3GPP TS 23.271 "Functional stage 2 description of LCS (Release 5)". June 2003.

[6] 3GPP2 S.R0066-0. "IP Based Location Services, Stage 1 Requirements". Version 1.0. April 2003.

[7] 3GPP TS 23.032. "Universal Geographical Area Description (GAD)".

[8] LIF TS 101. "Location Interoperability Forum (LIF) Mobile Location Protocol". June 2002.

[9] E. Guttman. "Service Location Protocol Modifications for IPv6". IETF RFC 3111 , May 2001. 